\title{
Validation of radiometric standards for the laboratory calibration of reflected-solar Earth observing satellite instruments
}

\author{
James J. Butler ${ }^{\mathrm{a}^{*}}$, B. Carol Johnson ${ }^{\mathrm{b}^{*}}$, Joseph P. Rice ${ }^{\mathrm{b}^{*}}$, Steven W. Brown ${ }^{\mathrm{b}^{*}}$, and Robert A. Barnes ${ }^{\mathrm{c}^{*}}$ \\ ${ }^{a}$ NASA Goddard Space Flight Center \\ ${ }^{b}$ National Institute of Standards and Technology \\ ${ }^{\mathrm{c}}$ Science Applications International Corporation
}

\begin{abstract}
Historically, the traceability of the laboratory calibration of Earth-observing satellite instruments to a primary radiometric reference scale (SI units) is the responsibility of each instrument builder. For the NASA Earth Observing System (EOS), a program has been developed using laboratory transfer radiometers, each with its own traceability to the primary radiance scale of a national metrology laboratory, to independently validate the radiances assigned to the laboratory sources of the instrument builders. The EOS Project Science Office also developed a validation program for the measurement of onboard diffuse reflecting plaques, which are also used as radiometric standards for Earth-observing satellite instruments. Summarized results of these validation campaigns, with an emphasis on the current state-of-the-art uncertainties in laboratory radiometric standards, will be presented. Future mission uncertainty requirements, and possible enhancements to the EOS validation program to ensure that those uncertainties can be met, will be presented.
\end{abstract}

Keywords: remote sensing, calibration, radiometry, radiance, reflectance

\section{INTRODUCTION}

Beginning in the 1980's NASA embarked on a highly successful series of Earth-observing satellite missions to determine how the land, ocean, water, and air are changing over time and to establish how much of these changes are of anthropogenic origin. Many of these changes have been found to be subtle and embedded in large natural variations. Detecting small systematic changes in the environment requires that successive series of satellite instruments be calibrated to unprecedented accuracies and all aspects of their optical, mechanical, thermal, and electrical operations be fully characterized. Achievement of the goals outlined for the U.S. Integrated Earth Observation System (IEOS) within the Global Earth Observation System of Systems (GEOSS) ${ }^{1,2}$ fundamentally requires that accurate and precise measurements of the Earth and Sun be acquired over the long term. Accurate pre-launch calibration, traceable to national radiometric standards, is mandatory and is the only way to ensure there is continuity in measurements from one flight system to the next with overlapping observations. Without traceability to standards, biases between measurement systems will lead to finite errors when calculating trends.

As Earth observation transitions from the U.S. NASA EOS-era to the international GEOSS-era, data sets obtained from satellite instruments flown by different national and international institutions will need to be merged. To ensure data quality and consistency, it is critically important that the sensors are calibrated against a common scale. That common scale is the internationally recognized International System of Units (SI). In addition to accurate pre-launch calibration and characterization, an integrated program for post-launch calibration and characterization is essential to ensure the accurate accounting of satellite instrument drift. Post-launch validation takes many forms and relies on on-board systems and comparison with ground, air, and balloon observations measuring the same parameters as the satellites. Comparisons are typically made between observed radiances (that is, vicarious calibration or level .1 validation) or between derived geophysical products. For long-term trend detection, comparisons of radiances are more desirable since the inversion algorithm is bypassed and the focus is on instrument sensitivity, which is most likely changing over time.

*(JJB, contact author) James.J.Butler@nasa.gov; phone 1301 614-5942; fax 1301 614-6695; Biospheric Sciences Branch, Code 614.4; NASA's Goddard Space Flight Center, Greenbelt, MD 20771 USA; *(BCJ) cjohnson@nist.gov; phone 1301 975-2322; fax 1 301 869-5700; Optical Technology Division, National Institute of Standards and Technology, Gaithersburg, MD 20899 USA; *(JPR) joe.rice@nist.gov; phone 1301 975-2133; fax 1301 869-5700; Optical Technology Division, National Institute of Standards and Technology, Gaithersburg, MD 20899 USA; *(SWB) steven.brown@nist.gov; phone 1 301 975-5167; fax 1 301 869-5700; Optical Technology Division, National Institute of Standards and Technology, Gaithersburg, MD 20899 USA; *(RAB) Robert.A.Barnes @nasa.gov; phone 1301 286-0501; fax 1301 286-0268; Science Applications International Corporation, 4600 Powder Mill Rd., Beltsville, MD 20705. 
Facilities which calibrate and characterize satellite and validation instrumentation must demonstrably establish and maintain traceability to the SI by executing traceability to radiometric standards disseminated by National Metrology Institutes (NMIs) such as the National Institute of Standards and Technology ${ }^{3,4}$. Traceability provides a means of establishing confidence in quantitative measurements by different sensors or measurements at different times. Evaluation and documentation of uncertainty in measurements is an integral part in establishing traceability ${ }^{5}$. Typically the uncertainties in a radiometric measurement are validated through comparisons with other independent laboratories and institutions. At the National Metrology Institute level, key measurement comparisons organized by the International Bureau of Weights and Measures (BIPM) under the Treaty of the Meter establish the degree of equivalence between national laboratories (for example, Lambe ${ }^{6}$ ).

\section{PREVIOUS TRANSFER RADIOMETER COMPARISONS}

In 1995, the EOS calibration program as part of the EOS Project Science Office (EOS) initiated a measurement assurance program to validate the radiance scales assigned to uniform sources used by satellite instrument builders and by vicarious calibration laboratories. For the visible to shortwave infrared wavelength range, those sources are internally and externally illuminated integrating spheres and diffuse reflectance panels illuminated by standard lamps. The assurance program employed a set of stable radiometers operating in the visible, near infrared, and shortwave infrared wavelength regions, which traveled to the instrument builder and vicarious calibration facilities to participate in radiometric measurement comparisons ${ }^{7}$. The radiometers have different, but well established traceabilities to primary standards. A description of each radiometer is given briefly below.

2.1. EOS VXR. The EOS Visible Transfer Radiometer (VXR), built in 1996 for the EOS Project Science Office, is a six channel filter radiometer similar in design to the SeaWiFS Transfer Radiometer (SXR) ${ }^{8}$. The VXR was designed and built by NIST for the EOS Project Science Office at NASA's GSFC. Both instruments use a camera lens to focus the object at the field stop. Behind the field stop, six wedge-shaped mirrors with spherical curvature focus the field stop at six image locations, where individual interference filters and silicon photodiodes are located. An on-axis optical system is used to align and focus the VXR. The field-of-view (FOV) of the VXR is $2.5^{\circ}$, and the minimum object distance is $85 \mathrm{~cm}$. The VXR filters were chosen to coincide with several visible and near-infrared bands of the MODIS, ASTER, and MISR instruments on the EOS Terra platform ${ }^{7}$. The VXR was calibrated against lamp-illuminated integrating sphere sources that were calibrated on NIST's Facility for Automated Spectral Irradiance and Radiance Calibrations (FASCAL).

2.2. EOS SWIXR. The EOS SWIXR is a scanning, double-grating monochromator-based instrument ${ }^{10}$. It is equipped with all-reflective input optics and a liquid-nitrogen-cooled indium antimonide (InSb) detector. The instrument has a full-angle field-of-view of $5.2^{\circ}$. A cold filter with transmittance less than $10^{-3}$ is placed in front of the InSb detector to reduce stray light and thermal infrared background radiation incident on the detector. Order sorting filters further reduce stray light in the system. The SWIXR has a stray light rejection of $10^{-7}$, a wavelength reproducibility of $0.1 \mathrm{~nm}$, and a corrected wavelength uncertainty of $0.2 \mathrm{~nm}$. Chopped output from the detector is sent to a three-stage trans-impedance amplifier and then to a lock-in amplifier. The instrument is calibrated using the NIST Portable Radiance (NPR) lamp-illuminated integrating sphere, which is calibrated for spectral radiance in FASCAL ${ }^{9}$. The operating wavelengths for the SWIXR range from $800 \mathrm{~nm}$ to $2500 \mathrm{~nm}$, with a variable bandwidth depending on the slit widths.

2.3. UA VNIR. The University of Arizona Visible/Near Infrared Radiometer (UA VNIR) is a single channel radiometer with a manually rotated filter wheel containing seven narrow-band interference filters. The UA VNIR is a sevenchannel filter radiometer with a detector employing three Si photodiodes arranged in a light-trapping configuration ${ }^{11}$. It was built and independently characterized by the UA Optical Sciences Center Remote Sensing Group. The optical design is based on two precision apertures separated a precisely known distance by Invar rods. The filters are placed in front of the first aperture and the trap detector is placed behind the second aperture. The UA VNIR channels were chosen to have center wavelengths corresponding to those in the Moderate Resolution Imaging Spectrometer (MODIS) and the Advanced Spaceborne Thermal Emission and Reflection Radiometer (ASTER). An eighth radiometer position is permanently shuttered to enable measurement of the radiometer dark signal. Traceabilty to NIST primary standards (and the SI) is established by calibrating the UA VNIR radiometer against standards of spectral irradiance. Two spectral irradiance standards were used for the calibration: one standard came directly from NIST (F-330) and one was obtained from a secondary standards laboratory (F-297). The standard in both cases is a quartz halogen lamp rated at $1000 \mathrm{~W}$ mounted in a special bi-post base with calibration traceable to the NIST 1990/1992 irradiance scale.

2.4. UA SWIR. The University of Arizona Shortwave Infrared Radiometer (UA SWIR) is a twelve-channel filter radiometer employing a liquid nitrogen cooled InSb detector ${ }^{12}$. Three channels have band-center wavelengths below 
$1 \mu \mathrm{m}$ and nine channels have center wavelengths beyond $1 \mu \mathrm{m}$. Similar to the UA VNIR, the UA SWIR uses two precision apertures to define the solid angle of its field of view. The UA SWIR channels are chosen to have center wavelengths corresponding with those of the MODIS and ASTER satellite instruments. Light incident on the radiometer is chopped, and the detector output is sent to a lock-in amplifier. To reduce noise and increase sensitivity, the InSb detector is operated at liquid nitrogen temperature $\left(77^{\circ} \mathrm{K}\right)$, along with the apertures, baffles, and amplifier. To further reduce unwanted thermal infrared background signal, a cold filter, also at liquid nitrogen temperature, is used to block radiation at wavelengths longer than $2.7 \mu \mathrm{m}$. Traceability is established by calibrating the UA SWIR radiometer for spectral radiance responsivity using standard irradiance lamps and a diffusely reflecting Spectralon panel. The lamps are the same as those used in the calibration of the UA VNIR instrument.

2.5. AIST. The National Institute of Advanced Industrial Science and Technology (AIST) ASTER radiometers are three single-filter radiometers built for the Japanese Observation System Organization (JAROS) with center wavelengths designed to correspond to the visible and near infrared wavelengths of the ASTER instrument ${ }^{13}$. The NLRM Ocean Color Temperature Scanner/Polarization and Directionality of Earth's Reflectances (OCTS/POLDER) are six singlefilter radiometers built for the National Space Development Agency (Japan) (NASDA) with center wavelengths and bandwidths close of those to the OCTS instrument ${ }^{14}$. These radiometers were calibrated using an AIST copper point blackbody source.

2.6. LXR. The Landsat Transfer Radiometer (LXR) ${ }^{15}$ is a six-filter radiometer that shares the design of the SXR, VXR, and SeaWiFS Transfer Radiometer II (SXR II) ${ }^{8}$. The LXR was built by NIST for the Landsat Project Science Office. The relative spectral responses for the first four LXR filter channels were configured to correspond to those for the Landsat ETM+ instrument ${ }^{16}$. The two narrow filter channels were chosen to match existing, similar channels in the VXR, SXR, and SXR II to facilitate comparisons between radiometers. Thus, there are four broad spectral response channels and two narrow ones, with the broad channels overlapping the narrow ones. The relative spectral response of the six LXR channels was determined using a lamp-illuminated monochromator at GSFC ${ }^{15}$. LXR traceability was established by having NIST calibrate the instrument.

2.7. SXR. The SeaWiFS Transfer Radiometer (SXR)is a six-channel filter radiometer built by NIST for the SeaWiFS Project at NASA GSFC ${ }^{8}$. It is the prototype for three other radiometers in the comparison campaigns: the VXR, LXR, and SXR II. For these comparisons, the SXR was calibrated for radiance against the OL420 integrating sphere source at NIST's FASCAL ${ }^{9}$.

2.8. SXR II. The SeaWiFS Transfer Radiometer II (SXR II) ${ }^{17}$ is a six-filter radiometer built by the SIMBIOS Project at NASA GSFC. It shares the basic design of the SXR, VXR, and LXR. The light is captured by a camera objective lens. Wedge shaped mirrors reflect the light to six detectors with filters for the respective band. The interference filters and silicon photodiode detectors are temperature controlled. The signal is recorded by an external voltmeter sequentially from channel 1 to 6 . The SXR-II was calibrated in yearly intervals from December 2000 to January 2003 at NIST's Spectral Irradiance and Radiance Calibration with Uniform Sources (SIRCUS) ${ }^{18}$.

2.9. 746/ISIC. NASA's GSFC maintains and operates an Optronic Laboratories Model 746, scanning, single-grating monochromator-based spectroradiometer equipped with a $10.2 \mathrm{~cm}$ diameter Integrating Sphere Irradiance Collector (ISIC) located at the monochromator entrance port ${ }^{19}$. In the visible/near infrared wavelength region from $400 \mathrm{~nm}$ to $1000 \mathrm{~nm}$, the 746/ISIC collects data in $10 \mathrm{~nm}$ steps, with an instrument bandwidth of $10 \mathrm{~nm}$. In the short-wave infrared spectral region from $1000 \mathrm{~nm}$ to $1600 \mathrm{~nm}$, data are obtained every $20 \mathrm{~nm}$ with a bandwidth of $20 \mathrm{~nm}$. In the wavelength region from $1600 \mathrm{~nm}$ to $2400 \mathrm{~nm}$, data are obtained every $20 \mathrm{~nm}$ with a $40 \mathrm{~nm}$ bandwidth. A silicon detector is used for the $400 \mathrm{~nm}$ to $1000 \mathrm{~nm}$ spectral range, while a lead sulfide detector is used for the $1000 \mathrm{~nm}$ to $2400 \mathrm{~nm}$ spectral range. The 746/ISIC transfers the irradiance calibration from a NIST-calibrated irradiance standard lamp to the integrating sphere being measured ${ }^{20}$.

2.10. Comparison Results. A portion of the results from seven instrument comparison campaigns ${ }^{7}$ are shown in Fig. 1. Each instrument has its own set of measurement wavelengths. The relative spectral responsivities of the instruments and the spectral radiances of the sources were used to calculate the band-averaged radiances for the purpose of comparing results. Many of the filter radiometers share several bands that are close in wavelength. Figure 1 shows results from three of those wavelengths, one in the blue, one in the near infrared, and one in the shortwave infrared. For each wavelength, the radiometers view the same source, and the results are shown as the percent difference of each radiometer's measurements from the average for the set of measurements. For panel $1 \mathrm{a}(442 \mathrm{~nm})$ the agreement among the measurements is $1.3 \%$, for panel $1 \mathrm{~b}(868 \mathrm{~nm})$ the agreement among the measurements is $1.3 \%$, and for panel $1 \mathrm{c}(1636$ $\mathrm{nm})$, the agreement is $2.5 \%$. 

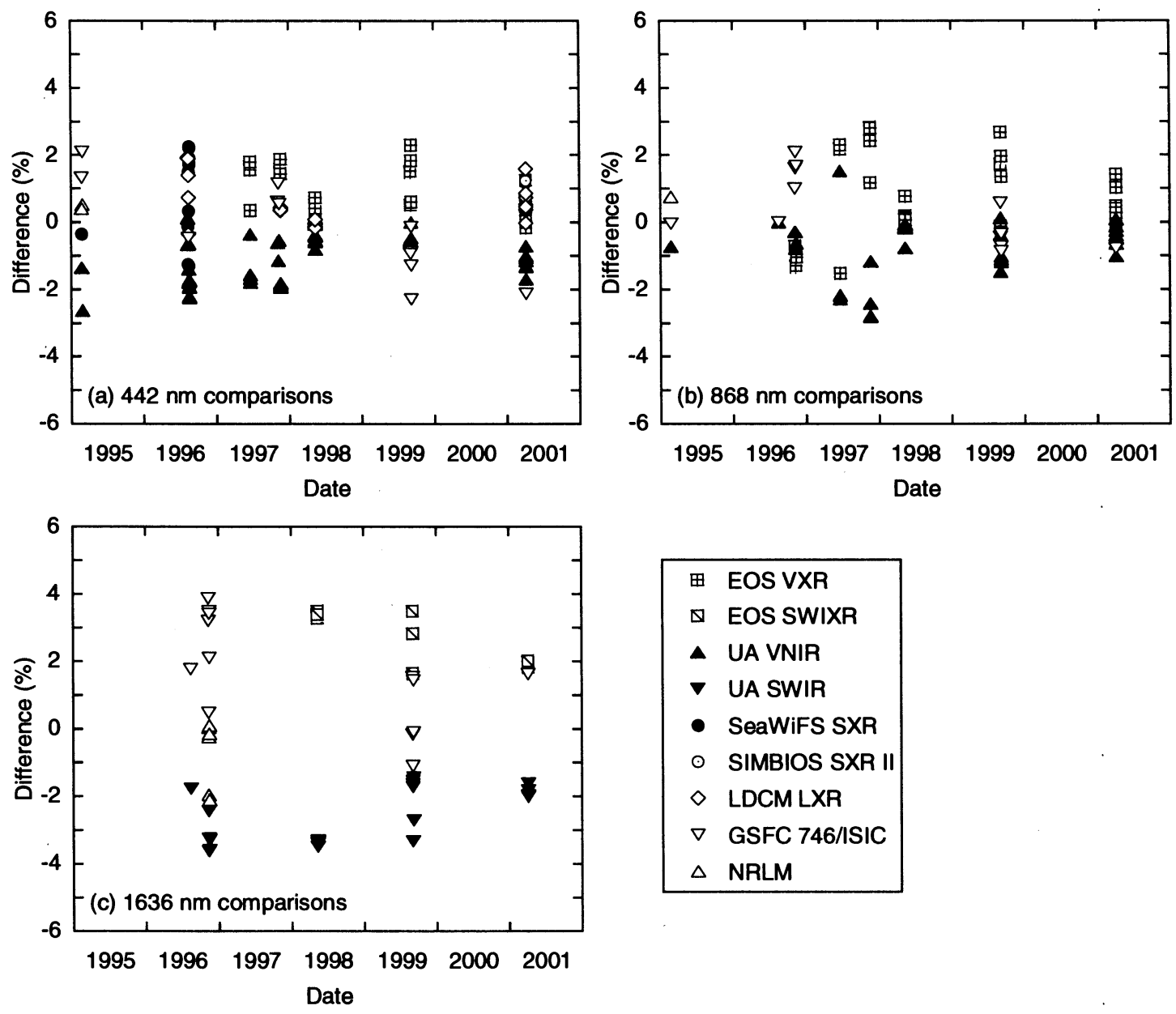

Fig. 1. Results from seven measurement campaigns ${ }^{7}$. The results are for three wavelengths, one in the visible, one in the near infrared, and one in the shortwave infrared. For each campaign and for each wavelength, these values have been normalized to the average for all measurements.

(a) Measurements at $442 \mathrm{~nm}$.

(b) Measurements at $868 \mathrm{~nm}$.

(c) Measurements at $1636 \mathrm{~nm}$.

Figure 2 shows a portion of the results from transfer radiometer measurements in 1998 of the integrating sphere source used to calibrate the Moderate Resolution Imaging Spectroradiometer (MODIS) and the Landsat 7 Enhanced Thematic Mapper Plus (ETM+ $)^{21}$. The results are for measurements of a single radiance level on a single day. The measurements are made by five of the transfer radiometers and show the percent differences of the radiometer radiances from those predicted from the calibration of the integrating sphere. The results in the panels include the estimated uncertainties for the measurements by each radiometer $(k=1)$. For this measurement campaign, the uncertainties for the EOS VXR were estimated to be $2 \%$ for all but the $870 \mathrm{~nm}$ channel for which the uncertainty was estimated to be $3 \%$. For this campaign the uncertainties for the UA VNIR were estimated to be $2.2 \%$. For the LXR the relative standard uncertainty of the measurements ranged from $2 \%$ to $3 \%$ depending on the channel. For the UA SWIR, the estimated uncertainties were estimated to be between $3.3 \%$ and 3.9\%. And for this measurement campaign the uncertainties for the EOS SWIXR were estimated to be $4.5 \%$. Overall, the agreement between the transfer radiometers falls within the estimated uncertainties.

Figure 3 shows a portion of the results from transfer radiometer measurement in 2001 of an integrating sphere source at NASA's GSFC ${ }^{19}$. Again the results are for measurements of a single radiance level on a single day, and the results 

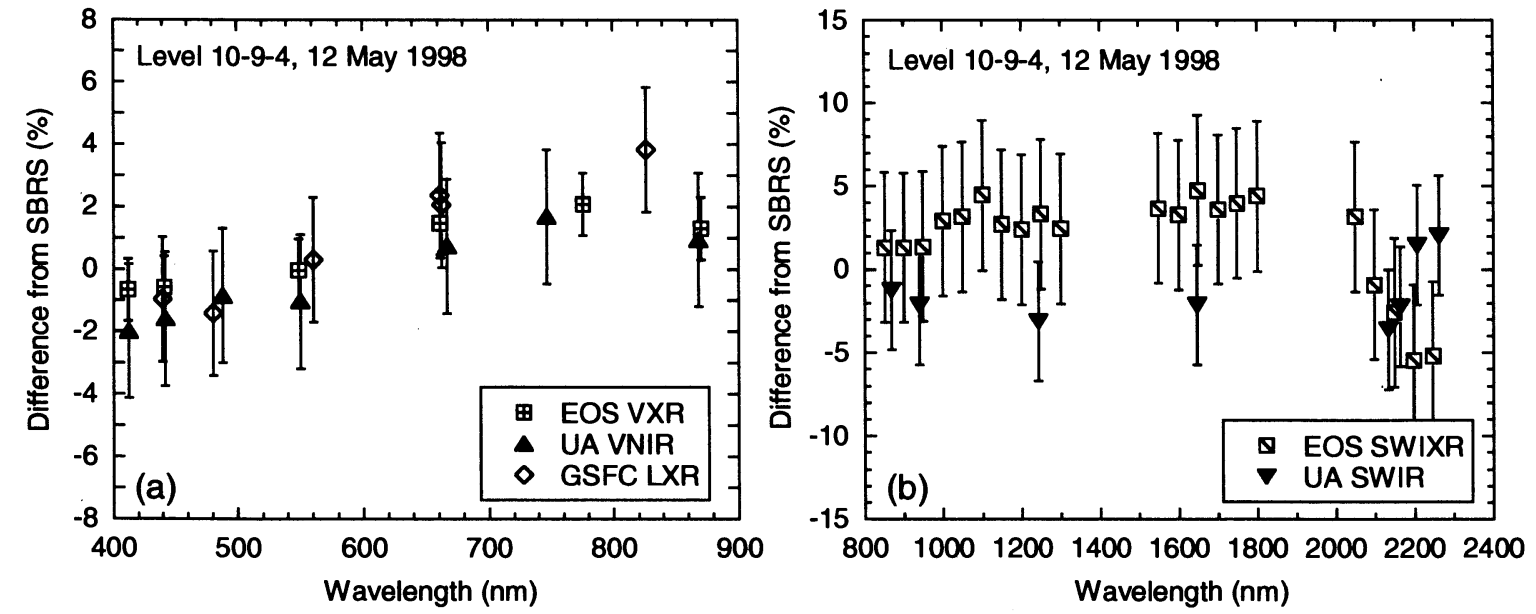

Fig. 2. Results from the measurement campaign at the radiance source for the calibration of MODIS and Landsat ETM+ (ref. 21). The results are for a single lamp setting of the source. The error bars are given for $k=1$ and reflect the estimated uncertainty for each instrument band at the time of the comparison.

(a) Measurements in the visible and near infrared.

(b) Measurements in the shortwave infrared.

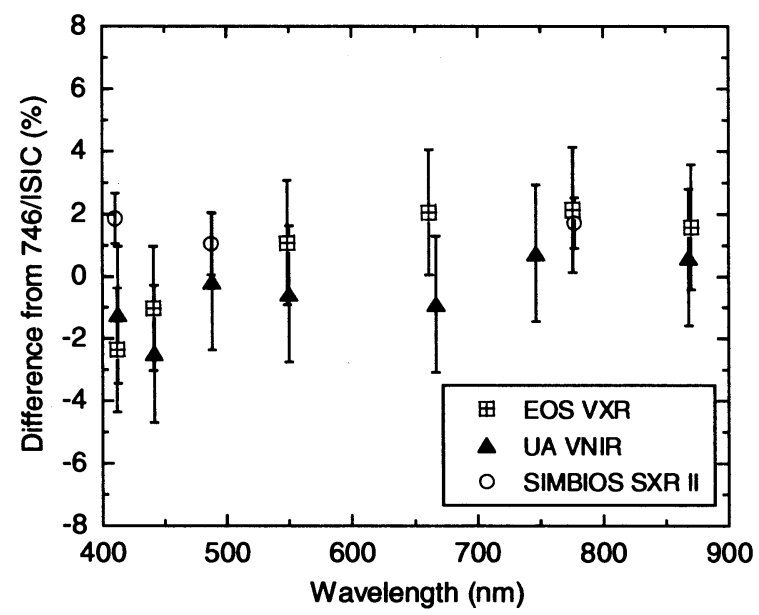

Fig. 3. Results from a transfer radiometer comparison at NASA's GSFC in $2001^{19}$. The error bars are given for $k=1$ and reflect the estimated uncertainty for each instrument band at the time of the comparison.

show the differences of the three radiometers from the radiance predicted from the calibration of the sphere. As with Fig. 2, the estimated uncertainties for radiometers range from about $2 \%$ to about $3 \%$. And as with Fig. 2, the overall agreement between the transfer radiometers falls within the estimated uncertainties.

For the measurement results presented here, the transfer radiometers are capable of validating the radiances from laboratory integrating sphere sources at the $2 \%$ to $3 \%$ level.

\section{PREVIOUS DIFFUSER MEASUREMENT COMPARISONS}

As a verification of the BRDF measurements related to EOS, the EOS PSO and NIST designed a round-robin experiment with four diffuse reflectors measured by five different laboratories ${ }^{22}$. The central facility in the round-robin is the Spectral Tri-function Automated Reference Reflectometer (STARR) at NIST ${ }^{23}$. Measurements were alternated between 

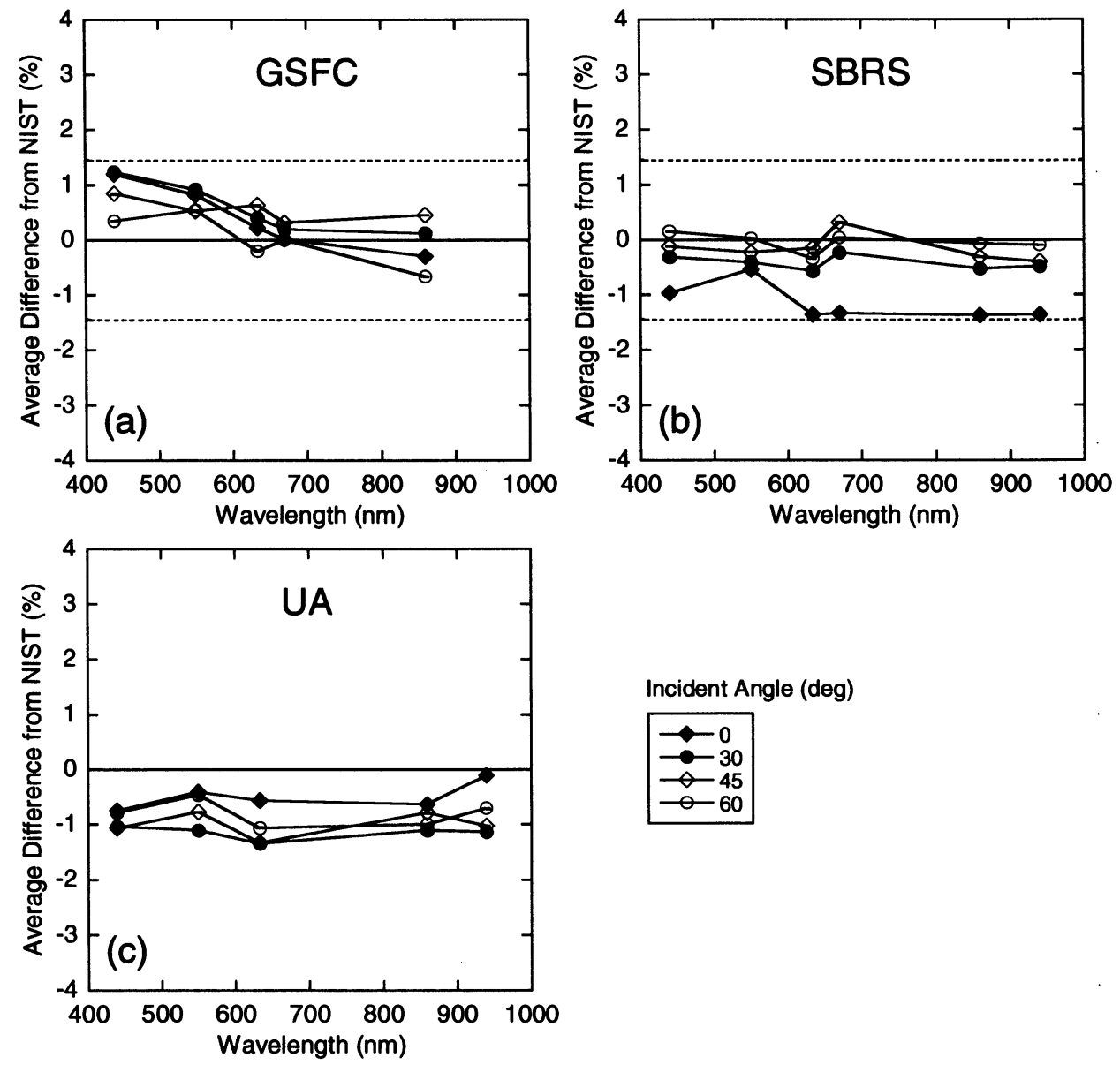

Fig. 4. Results from the reflectance round-robin in Early et al. ${ }^{22}$. The results give the percent difference of the measurements at three laboratories from those at NIST. The measured sample is Spectralon. The standard uncertainties for GSFC and SBRS (k=2) are shown as dotted lines. The standard uncertainty for the University of Arizona $(\mathrm{k}=2)$ is $4 \%$.

(a) Results for GSFC.

(b) Results for NIST.

(c) Results for the University of Arizona.

NIST and the other laboratories. Those laboratories were at the NASA GSFC, NASA JPL, Raytheon Santa Barbara Remote Sensing (SBRS), and the Remote Sensing Group of the Optical Sciences Center at the University of Arizona (UA). The four samples were Spectralon, pressed PTFE, sintered PTFE, and aluminum ${ }^{22}$.

Figure 4 shows measurements results for the Spectralon panel. Measurements were made at 440, 550, 633, 670, 860, and $940 \mathrm{~nm}$. Measurements were made at incident polar angles of $0,30,45$, and $60 \mathrm{deg}$., and at viewing polar angles from -60 to +60 deg in 10 deg steps. Figure 4 shows the results for Spectralon averaged over the set of viewing angles. The results are given as the average percent difference of the laboratory measurements from NIST. For GSFC and SBRS, the dotted lines give the standard uncertainties for the laboratory measurements $(k=2)$. For the University of Arizona, the standard uncertainty $(\mathrm{k}=2)$ is $2 \%$. For the results in Fig. 4, the agreement among the laboratories with NIST is at the $1.5 \%$ level. Overall, the experiment showed that the laboratories in the round-robin were able to make measurements within $2 \%$ of the values reported by NIST.

Subsequently, a reflectance round-robin was performed by three laboratories for measurements at wavelengths in the near ultraviolet ${ }^{2}$. These laboratories were NIST, GSFC, and the TPD TNO (formerly the TNO Institute of Applied Physics) in the Netherlands. The measurements were made at the standard wavelengths for the Total Ozone Mapping Spectrometer (TOMS) - 308.6, 312.5, 317.5, 322.2, 331.2, and $360.0 \mathrm{~nm}$ - and at the standard incident and viewing 


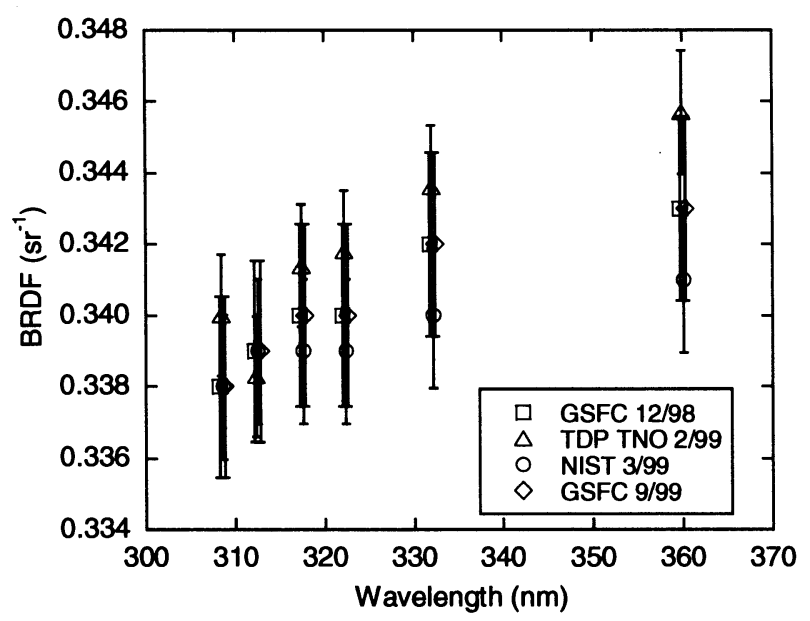

Fig. 5. Results from the reflectance round-robin in Butler et al. ${ }^{24}$. The results show the measured BRDF from each laboratory. The error bars give the standard uncertainty $(k=1)$ for each laboratory.

polar angles for the instrument -41 and 39 deg., respectively ${ }^{24}$. Figure 5 gives the results for one of the TOMS diffuser samples (SN 001). The measurement sequence among the three laboratories were GSFC (December 1998), TNO TPD (February 1999), NIST (March 1999), and GSFC (September 1999). The repeated measurements at GSFC were designed to give an indication of changes, if any, in the samples. Figure 5 also includes the standard uncertainty $(k=1)$ for each of the laboratories during the round-robin: $0.75 \%$ for GSFC, $0.5 \%$ for TPD TNO, and $0.6 \%$ for NIST. As shown in Fig. 5, the measurements at each wavelength agree to within the $k=1$ uncertainties. Overall, the agreement between the three laboratories was at the $1 \%$ level.

\section{DECREASING UNCERTAINTIES IN FUTURE TRANSFER RADIOMETER MEASUREMENTS}

For the measurement campaigns in Section 2, uncertainties of $2 \%$ to $3 \%$ in the transfer radiometer measurements of the uniform radiance sources used in the calibration of the satellite instruments were adequate for prelaunch calibration requirements for the instrument builders. For the EOS era, the requirements for the instrument system level pre-launch radiance calibration were set at the 4 to $5 \%$ level, of which source calibration uncertainty was the dominant term. For future instruments with improved capabilities, these calibration and characterization requirements will become more stringent. As a result, improvements in the uncertainties for the transfer radiometers will be necessary. A substantial portion of this improvement can come from better characterization of the radiometers.

4.1. Monitoring Radiometer Changes Between Calibrations. Recently developed calibration facilities, such as the Spectral Irradiance and Radiance Calibration with Uniform Sources (SIRCUS) facility at NIST, allow radiometer calibrations at the sub-percent level. However, the calibration of a transfer radiometer at SIRCUS has little value unless the stability of the radiometer's measurements over time has been determined. Routine calibrations of an individual radiometer at a primary calibration facility several times per year is impractical and time consuming. In contrast, measurements of a diffuse light source, such as an integrating sphere or a lamp/plaque can be done on a routine basis. In most cases, a dedicated test fixture should be practical to be devised, that is, a fixture that allows reproducible positioning of the radiometer relative to the test source. In addition, reproducible operating conditions for the source (lamp current, or currents), plus reproducible ambient conditions (temperature, for example), should be established to provide a consistent flux reference for the radiometer over time. Of course, the outputs of spheres or lamp/plaque arrangements do change over time as the lamps and diffuse surfaces age. Except for lamp failures, these changes are expected to occur slowly over time causing a decrease in the radiance from the source. Experience has shown that these changes occur more rapidly at shorter wavelengths, so the flux from the sources tends to become more "yellow."

An example of long-term measurements of an integrating sphere by a transfer radiometer is shown in Fig. 6 . In this example, the radiometer is the EOS VXR and the spheres are the NIST Portable Radiance (NPR) source ${ }^{25}$ and the NIST Portable Radiance 2 source (NPR2), which is a copy of the NPR. The figure gives the signals from the VXR $870 \mathrm{~nm}$ band after normalization. For each sphere source, the signals are normalized to the average for the set of measurements. Potential variability in the radiances from the spheres are not considered in these time series. For the measurements of 


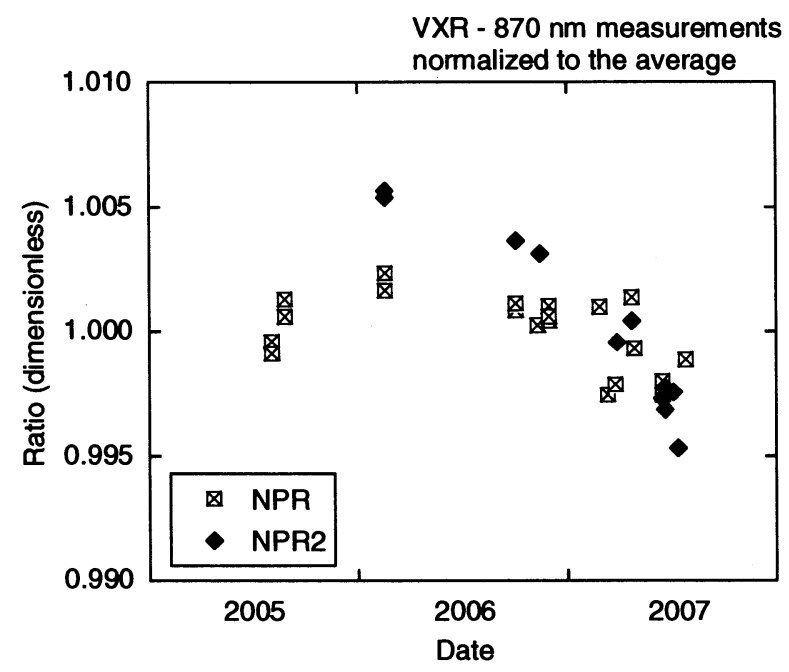

Fig. 6. Measurements of the NPR and NPR2 spherical integrating sources by the EOS VXR. For each source, the signals from the VXR are normalized to the average value for the set of measurements. The range of the vertical scale is $\pm 1 \%$. The changes in the measurements come from changes in the sources as well as the radiometer. For the purpose of monitoring changes in the radiometer, these data are sparse, since possible changes in the data gaps are not shown.

the NPR, the maximum difference in the VXR signals is one-half percent over 24 months. For the measurements of the NPR2, the maximum difference is one percent over 18 months.

For the NPR measurements in late 2006 and early 2007, the scatter in the results is around one-half percent. This scatter is a substantial portion of an uncertainty budget at the $1 \%$ level. Unfortunately, the sparseness of the data set does not allow a better characterization of the scatter. For the tracking of transfer radiometer changes to be effective, sphere measurements must be made routinely and on a regular schedule - once a week, on the same day each week. The routine and regular basis for these measurements will create a consistent time series. The time series suggested here will not resolve problems in the routine measurements, such as the scatter in the NPR measurements in 2006/2007 or the change in the NPR2 measurements over the same time period. These will need to be examined independently.

The time series is only part of a program that includes regular calibrations of the transfer radiometer and the sphere, on an annual basis. The calibrations will allow resolution of the source of the long-term changes in the sphere measurements. And the long-term sphere measurements will give an understanding of the changes in the transfer radiometer between calibrations. Intermittent calibrations of these radiometers are inadequate to track their changes over time $\mathrm{e}^{26}$. They must be performed on a routine basis as well.

4.2. Mapping Radiometer Fields-of-View. The measurement equation for a transfer radiometer ${ }^{27,28}$ contains terms for both the spectral and spatial response of the radiometer. In general, the relative spectral responses for the transfer radiometers are well determined, both in the in-band and out-of-band regions. However for most of these instruments, the relative spatial response is not that well determined. For one of the instruments in Section 2, the SXR, the relative spectral response has been determined and documented ${ }^{8}$. At the $1 \%$ level, knowledge of the spatial response becomes important since the outputs of the spheres are not necessarily constant across their apertures.

4.3. Mapping Sphere Apertures. Since different transfer radiometers have different fields of view, it is important to map the output of the integrating spheres at the instrument vendors and the vicarious calibration facilities. A map of the aperture of the NIST Portable Radiometer (NPR) is shown in Brown and Johnson ${ }^{25}$. The actual properties of the radiometers and the source under study contribute to the measurement equations. These properties can contribute to instrument-to-instrument differences in the results from the measurement campaigns.

4.4. Wavelength Interpolation. The interpolation in the spectral radiance (or irradiance) reference values and the spectral responsivity values for the transfer radiometers to a common wavelength interval for the purpose of determining band-averaged radiances should be considered in more detail. The calibration values in spectral radiance are sparse, with separations as much as $50 \mathrm{~nm}$, and spectral features cannot be captured with analytical fits such as cubic spline or 
linear interpolation. In addition, because the underlying values have uncertainty, it is questionable to select methods such as cubic spline that force agreement with the calibration value. For the spectral responsivity data, spectral radiance(or irradiance) values must be acquired at the system level over the full spectral range over which the detector has response, again with sufficient resolution to accurately represent the instrument's responsivity.

\section{EXPANDING DIFFUSER BRDF MEASUREMENT CAPABILITIES TO BETTER SUPPORT ON-ORBIT REFLECTANCE CALIBRATIONS}

5.1. Shortwave Infrared BRDF Measurements. The BRDF measurement capabilities of the NIST Spectral Trifunction Automated Reference Reflectometer (STARR) facility and many other secondary measurement facilities must be expanded to include bi-directional measurements over the $1000 \mathrm{~nm}$ to $2500 \mathrm{~nm}$ spectral interval. Users require laboratory measurements of BRDF to uncertainties of $1 \%$ or better $(\mathrm{k}=1)$, to meet typical system level and on orbit reflectance measurement requirements of $2 \%$ or better ${ }^{29}$ across the entire reflected solar wavelength region. High accuracy BRDF measurements in the shortwave infrared are more challenging than those in the visible and near infrared. This is partially due to the need to use cooled detectors of known linearity to work in this region and the lower incident and hence reflected light levels realized. From 400 to $900 \mathrm{~nm}$, the uncertainty of BRDF measurements from the NIST STARR facility on Spectralon samples is better than $0.6 \%(\mathrm{k}=2)$ for scatter angles up to $\pm 60^{\circ}$ (NIST BRDF Measurement Report Test Number 844/271744, April 26, 2005) and meets the user BRDF specification in this wavelength region. Since many secondary laboratories employ NIST measured diffuse targets in a relative BRDF measurement approach, uncertainties in BRDF from these standards laboratories will be greater than the NIST uncertainty. However, this apparently good news concerning low BRDF measurement uncertainties in the visible and near infrared does not satisfy the real requirement for similarly high quality BRDF measurements in the shortwave infrared.

5.2. Goniometric Limitations of BRDF Measurement. Earth observing instruments deploy solar illuminated diffuse targets to monitor on-orbit radiance responsivity over the solar reflective wavelength region. For any given satellite instrument, the range of angles of on-orbit illumination of the target by the Sun usually does not lie in the same plane as the angle at which the instrument views the target. Since no diffuse target is a perfect Lambertian scatterer, measurement of the BRDF over the geometrical range of out of plane incident and scatter angles experienced on-orbit is recommended. Depending on the solar illumination/instrument viewing relative geometries and the surface versus bulk scattering properties of the diffuse target, the deviation from the perfect Lambertian scattering case can be significant. This leads to the requirement for goniometers which can position the incident light and scatter detector at any set of angles above the scattering hemisphere of the diffuse target.

5.3. Sample Size and Sample Uniformity Limitations of BRDF Measurements. On-board diffuse targets vary widely in size and, by inference, weight. For example, the roughened aluminum flight diffusers used by the ultraviolet Total Ozone Mapping Spectrometer (TOMS) FM2 were on the order of $25 \mathrm{~mm}$ by $18 \mathrm{~mm}$, while the largest dimension of the YB-71 painted flight diffuser for the Landsat ETM+ instrument was on the order of $530 \mathrm{~mm}$. In order to meet the BRDF measurement requirements on flight diffusers, goniometric sample stages must be appropriately designed to accommodate a wide range of mounting adapters. In addition, BRDF measurement facilities such as STARR and the GSFC Diffuser Calibration Laboratory (DCL) illuminate diffuser areas which are typically smaller than the areas viewed by satellite instruments. In the absence of diffuser reflectance non-uniformities, this is not an issue. However, depending on the material, diffusers may exhibit significant spatial reflectance non-uniformities which must be measured over the field of view of the satellite instrument. An $x, y$ raster or uniformity reflectance imaging capability in a scatterometer are required to enable these measurements to be made.

5.4. Sample Shipping, Storage, and Handling. Contamination of diffuse target surfaces during shipping, storage, measurement, or handling directly impacts target reflectance and must be avoided in intercomparing and validating BRDF measurements between metrology laboratories at the $1 \%$ level. A proven technique for shipping diffuse targets while eliminating contamination was developed by the TOMS project. The TOMS project successfully employed a metal box with an o-ring edge seal and one-way vent valve to ship their diffusers between calibration facilities. Three Spectralon diffusers were securely mounted in the box with no surfaces in contact with the optically diffuse sides of the targets. When not in use or being measured, diffuse targets should be stored in clean sealed boxes or containers made from non-outgassing material. Measurement and deployment of targets should take place in a cleanroom environment. Handling of exposed diffuse targets should be performed in a cleanroom environment using gloves which do not emit particulates or leach hydrocarbons. Adherence to the above procedures will be necessary to achieve validated BRDF measurements at the sub $1 \%$ level. 


\section{ENHANCEMENTS TO THE EOS CALIBRATION/VALIDATION PROGRAM IN AN EFFORT TO MEET FUTURE REMOTE SENSING REQUIREMENTS}

Understanding the processes behind global climate change involves the measurement of small changes in optical signals from the Earth using instrumentation with low measurement uncertainties and high stabilities ${ }^{30}$. The initial and only opportunity to determine the measurement uncertainty and stability of an Earth observing satellite instrument under controlled, hands-on, conditions is during its pre-launch calibration and characterization in the cleanroom. The EOS calibration program attempted to address the pre-launch measurement uncertainty issue through series of radiometric measurement comparisons and reflectance round-robins involving instrument builder and validation facilities. These campaigns not only validated the radiometric scales and measurement capabilities of these facilities but also were most effective in enabling the peer measurement community to review and suggest improvements in the measurement methodologies of the participating institutions.

According to Ohring, et al. ${ }^{30}$, the required accuracies for radiance and reflectance measurements of the Earth in the visible through shortwave infrared for certain geophysical products are as low as $2 \%$. In the EOS integrating sphere measurement comparisons and reflectance round robins, integrating sphere radiances and diffuse target reflectances were validated to approximately that level, meaning that the uncertainty budgets for those geophysical products were largely consumed by the ability to calibrate and validate the sources and targets. Additional uncertainty terms cause the target measurement uncertainties to be exceeded.

Based upon the EOS experience, advancements or enhancements in pre-launch satellite instrument calibration and characterization will be realized through improvements in testing instrumentation and measurement methodologies which ultimately lead to better absolute standards and more fully account for sensor characterization issues. By leveraging on the significant database of historical radiance and monitor housekeeping measurements of the NPR integrating sphere, improvements to the general design and operation of integrating spheres are possible. Likewise, similar improvements could be realized in the setup and measurement of lamp-illuminated panels, a commonly used radiance calibration source. For the next generation of remote sensing instruments employing two-dimensional, multi-detector focal plane arrays, new calibration and characterization hardware approaches and techniques will be necessary to characterize and understand their optical performance. In response to the often relaxed requirement of calibrating a satellite instrument "as it flies using representative scenes," NIST is coupling spectrally tunable light sources with spatially programmable projection systems to produce a hyperspectral image projector (HIP) $)^{31,32,33}$. The HIP produces complex spectral, high fidelity spatial scenes similar to those remotely sensed by a satellite instrument ${ }^{34}$. In support of the calibration of reflectance-based instruments, NIST is also developing improved laser based measurement techniques to perform the system-level calibration and characterization of on-board diffuse reflectance systems ${ }^{35}$. A long-standing issue which has challenged many remote sensing instrument programs is radiance calibration in the region of the 1.38 micrometer water vapor absorption band. The complete but potentially expensive solution to this problem is to design and calibrate uniform radiance sources and radiometers for operating in vacuum.

\section{CONCLUSION}

Target radiance and reflectance calibration uncertainties for the EOS satellite instruments operating in the visible through shortwave infrared wavelength region were 4 to $5 \%$ and $2 \%(\mathrm{k}=1)$, respectively. The EOS radiometric measurement comparisons validated the calibration of the integrating sphere sources used in satellite instrument calibrations to the $\pm 3 \%$ level in the visible and near infrared and to the $\pm 5 \%$ level in the shortwave infrared. The EOS reflectance measurement round robins validated the calibration of diffuse calibration targets to the $\pm 2 \%$ level. The radiance and reflectance measurements of all participating facilities were within their combined measurement uncertainties. The radiometric and reflectance measurement comparison results reveal that for specific wavelengths, radiance levels, and reflectance geometries, a significant portion of the instruments' system level radiance and reflectance calibration uncertainty budgets are consumed by uncertainty in the calibration of sources and artifacts. In the post-EOS era, the calibration uncertainties of sources and artifacts will decrease through a series of near and long-term improvements in testing instrumentation and measurement methodologies. For the radiance calibration and validation of integrating sphere sources, this includes long-term monitoring of transfer radiometer responsivity changes between integrating sphere calibration deployments, mapping of radiometer fields-of-view, mapping of sphere aperture radiance uniformity, and uniformity in the interpolation of radiance/irradiance reference values and radiometer spectral responsivity. For reflectance calibrations, this includes: implementation of a low uncertainty BRDF measurement capability through the shortwave infrared; implementation of full out-of-plane and BRDF uniformity measurement capabilities, particularly in those facilities making measurements on flight articles; implementation of sample stages capable of handling a wide variety of 
sizes, shapes, and weights of diffuse on-board targets; and implementation of protocols to eliminate contamination of diffuse targets. For the next generation of remote sensing instruments employing two-dimensional, multi-detector focal plane arrays, new calibration and characterization hardware approaches and techniques will be necessary to characterize and understand their optical performance. In accord with the recommendation to calibrate an instrument "as it flies using representative scenes," NIST is developing an advanced radiance calibration source capable of producing complex spectral, high fidelity spatial scenes similar to those remotely sensed by a satellite instrument. In support of the calibration of reflectance-based instruments, NIST is also developing improved laser based measurement techniques to perform the system-level calibration and characterization of on-board diffuse reflectance systems.

\section{REFERENCES}

1. CENR/IWGEO, Strategic Plan for the U.S. Integrated Earth Observation System, Asrar, G., C. Gabriel, and G. Withee, co-chairs, (National Science and Technology Council Committee on Environment and Natural Resources, Washington, DC, 2005) (http//usgeo.gov/docs/EOCStrategic_plan.pdf).

2. GEO, Global Earth Observing System of Systems (GEOSS), Mitsos, A., T. Shirakawa, R. Adam, and C.C. Lautenbacher, co-chairs (Group on Earth Observations, Geneva, 2005) (http://www.earthobservations.org/doc_library/ summit_docs.html see Reference Document)

3. B. C. Johnson, S. W. Brown, and J. P. Rice, "Metrology for remote sensing radiometry," in Post-Launch Calibration of Satellite Sensors, ISPRS Book Series vol. 2, S. Morain, A. Budge eds. (A. A. Balkema Publishers, London, 2004), pp. 7-16.

4. J. P. Rice, and B. C. Johnson, "NIST activities in support of space-based radiometric remote sensing,: in Harnessing Light: Optical Science and Metrology at NIST, C. Londoño, ed., Proc. SPIE 4450 108-126 (2001).

5. J. J. Butler, B. C. Johnson, and R. A. Barnes, "The calibration and characterization of Earth remote sensing and environmental monitoring instruments," in Optical Radiometry, Experimental Methods in the Physical Sciences vol. 41, A. C. Parr, R. U. Datla, and J. L. Gardner, eds. (Elsevier Academic Press, San Diego, 2005), Chapter 10.

6. R. P. Lambe, R. Saunders, C. Gibson, J. Hollandt, and E. Tegeler, "A CCPR international comparison of spectral radiance measurements in the air-ultraviolet," Metrologia 37 51-54 (2000).

7. J. J. Butler, and R. A. Barnes, "The use of transfer radiometers in validating the visible through shortwave infrared calibrations of radiance sources used by instruments in NASA's Earth Observing System," Metrologia 40 S70-S77 (2003).

8. B. C. Johnson, J. B. Fowler, and C. L. Cromer, The SeaWiFS Transfer Radiometer (SXR), NASA Tech Memo. 1998-206892, Vol. 1, S. B. Hooker and E. R. Firestone, eds. (NASA Goddard Space Flight Center, Greenbelt, Md., 1998).

9. J. H. Walker, R. D. Saunders, and A. T. Hattenberg, Spectral Radiance Calibrations. NBS Special Publication, SP250-1 (U. S. Government Printing Office, Washington, D.C., 1987).

10. S. W. Brown, B. C. Johnson, and H. W. Yoon, "Description of a portable spectroradiometer to verify EOS radiance scales in the short-wave infrared, Earth Observer 10 43-44 (1998).

11. S. F. Biggar, and P. N. Slater, "Preflight cross-calibration radiometers for EOS AM-1 platform visible and near-ir sources, in Sensor Systems for the Early Earth Observing System Platforms," W. L. Barnes, ed., Proc. SPIE 1939 243-249 (1993).

12. P. R. Spyak, D. S. Smith, J. Thiry, and C. Burkhart, "Short-wave infrared transfer radiometer for the calibration of the Moderate-Resolution Imaging Spectrometer and the Advance Spaceborne Thermal Emission and Reflection Radiometer," Appl. Opt. 39 5694-5706 (2000).

13. F. Sakuma, M. Kobayashi, and A. Ohno, "ASTER round-robin radiometers for the preflight cross-calibration of EOS AM-1 instruments," Proc. IGARSS, 1995-1997 (1994).

14. F. Sakuma, T. Bret-Dibat, H. Sakate, A. Ohno, J. Perbos, J. M. Martinuzzi, K. Imako, H. Oaku, T. Moriyama, Y. Miyachi, and Y. Tange, "POLDER-OCTS preflight cross-calibration experiment using round-robin radiometers," in Infrared Spaceborne Remote Sensing III, M. Strojnik, and B. F. Andresen, eds., Proc. SPIE 2553 232-243 (1995).

15. B. L. Markham, J. S. Schafer, F. M. Wood, Jr., P. W. Dabney, and J. L. Barker, "Monitoring large-aperture spherical integrating sources with a portable radiometer during satellite instrument calibration," Metrologia 35 643648 (1988).

16. B. L. Markham, W. C. Boncyk, D. L. Helder, and J. L. Barker, "Landsat-7 Enhanced Thematic Mapper Plus radiometric calibration," Can. J. Remote Sens. 23 318-332 (1997).

17. G. Meister, P. Abel, R. Barnes, J. Cooper, C. Davis, M. Godin, D. Goebel, G. Fargion, R. Frouin, D. Korwan, R. Maffione, C. McClain, S. McLean, D. Menzies, A. Poteau, J. Robertson, J. Sherman, The First SIMBIOS Radiomet- 
ric Intercomparison (SIMRIC-1), April-September 2001, NASA Tech.Memo.2002-210006, (Goddard Space Flight Center, Greenbelt, Md., 2002).

18. S. W. Brown, G. P. Eppeldauer, K. R. Lykke, "NIST facility for spectral irradiance and radiance responsivity calibrations with uniform sources," Metrologia 37 579-582 (2000).

19. R. A. Barnes, J. W. Cooper, J. E. Marketon, S. W. Brown, B. C. Johnson, and J. J. Butler, "Bandwidth and spectral stray light effects in the NASA GSFC Radiometric Calibration Facility primary transfer radiometer," in Earth Observing Systems XI, J. J. Butler and J. Xiong, eds., Proc. SPIE 6296, 6296-04 (2006).

20. J. H. Walker, C. L. Cromer, and J. T. McLean, "A technique for improving the calibration of large-area sphere sources," in Calibration of Passive Remote Observing Optical and Microwave Instrumentation, B. W. Guenther, ed., Proc. SPIE 1493 224-230 (1991).

21. J. J. Butler, B. C. Johnson, S. W. Brown, R. D. Saunders, S. F. Biggar, E. F. Zalewski, B. L. Markham, P. N. Gracey, J. B. Young, and R. A. Barnes, "Radiometric Measurement Comparison on the Integrating Sphere Source Used in the Calibration of the Moderate Resolution Imaging Spectroradiometer (MODIS) and the Landsat 7 Enhanced Thematic Mapper (ETM $\left.{ }^{+}\right)$," J. Res. Natl. Inst. Stnds. and Technol. 108 199-208 (2003).

22. E. A. Early, P. Y. Barnes, B. C. Johnson, J. J. Butler, C. J. Bruegge, S. F. Biggar, P. R. Spyak, and M. M. Pavlov, "Bidirectional reflectance round-robin in support of the Earth Observing System program," J. Atmos. Oceanic. Technol. 17 1077-1091 (2000).

23. J. E. Proctor, and P. Y. Barnes, "NIST High Accuracy Reference Reflectometer-Spectrophotometer," J. Res. Natl. Inst. Stand. Technol., 101, 619-627 (1996).

24. J. J. Butler, H. Park, P. Y. Barnes, E. A. Early, C. van Eijk-Olij, A. E. Zoutman, S. van Buller-Leeuwen, and J. G. Schaarsberg, "Comparison of ultraviolet Bi-directional Reflectance Distribution Function (BRDF) measurements of diffusers used in the calibration of the Total Ozone Mapping Spectrometer (TOMS)," in Sensors, Systems, and Next-Generation Satellites VI, H. Fujisada, J. J. Lurie, M. L. Aten, and K. Weber, eds., Proc. SPIE 4881 345-354 (2003).

25. S. W. Brown, and B. C. Johnson, "Development of a portable integrating sphere source for the Earth Observing System's calibration validation programme," Int. J. Remote. Sens., 24, 215-224 (2003).

26. B. C. Johnson, S. W. Brown, and H. W. Yoon, "Radiometric calibration history of visible and near infrared transfer radiometers," Metrologia 37 423-426 (2000).

27. H. J. Kostlowski, and F. E. Nicodemus, "An introduction to the measurement equation," in Self-Study Manual on Optical Radiation Measurements, Part 1 - Concepts, F. E. Nicodemus, ed., NBS Tech. Note 910-2 (U.S. Department of Commerce, National Institute of Standards and Technology, Washington, DC, 1978) pp. 58-104.

28. B. C. Johnson, S. S. Bruce, E. A. Early, J. M. Houston, T. R. O'Brian, A. Thompson, S. B. Hooker, and J. L. Mueller, The Fourth SeaWiFS Intercalibration Round-Robin Experiment, SIRREX-4, May 1995, NASA Tech. Memo. 104566, Vol. 37, S. B. Hooker and E. R. Firestone, eds. (NASA Goddard Space Flight Center, Greenbelt, Md., 1996).

29. M. M. Pavlov, M. L. Byers, and J. A. Walker, "Bidirectional reflectance factor (BRF) characterization of the MODIS flight diffuser," in Earth Observing Systems III, W.L. Barnes, ed., Proc. SPIE 3439 257-268 (1998).

30. G. B. Ohring, B. Weilicki, R. Spencer, B. Emory, and R. Datla, Satellite Instrument Calibration for Measuring Global Climate Change, NISTIR 4047 (Nat. Inst. Stand. Technol., Gaithersburg, Md., 2004).

31. S. W. Brown, J. P. Rice, J. E. Neira, R. Bousquet, and B. C. Johnson, "Hyperspectral image projector for advanced sensor characterization," in Earth Observing Systems XI, J. J. Butler and J. Xiong, eds., Proc. SPIE 6296, 6296-02 (2006).

32. S. W. Brown, J. P. Rice, J. E. Neira, and B. C. Johnson, "Spectrally tunable sources for advanced radiometric applications," J. Res. Natl. Inst. Stnds, and Technol. 111 401-410 (2006).

33. J. P. Rice, S. W. Brown, B. C. Johnson, and J. E. Neira, "Hyperspectral image projectors for radiometric applications, Metrologia 43 S61-S65 (2006).

34. S. W. Brown, B. Myers, R. A. Barnes, and J. P. Rice, "Characterization of Earth observing satellites for response to spectrally and spatially variable scenes," in Earth Observing Systems XII, J. J. Butler and X. Xiong, eds., Proc. SPIE 6699 these proceedings (2007).

35. R. A. Barnes, S. W. Brown, K. R. Lykke, G. T. Fraser, and J. J. Butler, "System level pre-launch calibration of onboard solar diffusers," in Earth Observing Systems XII, J. J. Butler and X. Xiong, eds., Proc. SPIE 6699 these proceedings (2007). 